\title{
Article
}

\section{The Well Posedness for Nonhomogeneous Boussinesq Equations}

\author{
Yan Liu ${ }^{1}$ and Baiping Ouyang ${ }^{2, *}$ \\ 1 Department of Mathematics, Guangdong University of Finance, Guangzhou 510521, China; \\ 26-046@gduf.edu.cn \\ 2 School of Data Science, Guangzhou Huashang College, Guangzhou 511300, China \\ * Correspondence: oytengfei79@gdhsc.edu.cn
}

check for

updates

Citation: Liu, Y.; Ouyang, B. The Well Posedness for Nonhomogeneous Boussinesq Equations. Symmetry 2021, 13, 2110. https://doi.org/10.3390/ sym 13112110

Academic Editors: Octav Olteanu and Savin Treanta

Received: 19 October 2021

Accepted: 30 October 2021

Published: 6 November 2021

Publisher's Note: MDPI stays neutral with regard to jurisdictional claims in published maps and institutional affiliations.

Copyright: (c) 2021 by the authors. Licensee MDPI, Basel, Switzerland. This article is an open access article distributed under the terms and conditions of the Creative Commons Attribution (CC BY) license (https:// creativecommons.org/licenses/by/ $4.0 /)$.

\begin{abstract}
This paper is devoted to studying the Cauchy problem for non-homogeneous Boussinesq equations. We built the results on the critical Besov spaces $(\theta, u) \in L_{T}^{\infty}\left(\dot{B}_{p, 1}^{N / p}\right) \times L_{T}^{\infty}\left(\dot{B}_{p, 1}^{N / p-1}\right) \cap L_{T}^{1}\left(\dot{B}_{p, 1}^{N / p+1}\right)$ with $1<p<2 N$. We proved the global existence of the solution when the initial velocity is small with respect to the viscosity, as well as the initial temperature approaches a positive constant. Furthermore, we proved the uniqueness for $1<p \leq N$. Our results can been seen as a version of symmetry in Besov space for the Boussinesq equations.
\end{abstract}

Keywords: non homogenous boussinesq equations; global well-posedness; littlewood-paley decomposition

\section{Introduction}

This paper discusses the global well-posedness of Boussinesq equations. We assume that the viscosity and thermal conductivity are temperature dependent. The coupled mass flow and heat flow of the viscous incompressible fluid are controlled by Boussinesq approximation. The equations we study are as follows:

$$
\left\{\begin{array}{l}
u_{t}-\operatorname{div}(v(\theta) \nabla u)+u \cdot \nabla u+\alpha \theta g+\nabla p=0 \\
\operatorname{div}(u)=0 \\
\theta_{t}-\operatorname{div}(\kappa(\theta) \nabla \theta)+u \cdot \nabla \theta=0
\end{array}\right.
$$

Here $u(t, x)$ denotes the velocity of the fluid, $(t, x) \in R^{+} \times R^{N}, N \geq 2$ is the spatial dimension; $p(t, x)$ is the hydrostatic pressure; $\theta(t, x)$ is the temperature; $g(t, x)$ is the external force by a unit of mass; $v(\theta)$ is the kinematic viscosity; $\kappa(\theta)$ is the thermal conductivity; $\alpha$ is a positive constant which is dependent on the coefficient of volume expansion. The Boussinesq system has important roles in the atmospheric sciences, for more details, one could refer to $[1,2]$.

The homogeneous Boussinesq equations corresponding to the special case where coefficients $v$ and $\kappa$ are positive constants:

$$
\left\{\begin{array}{l}
u_{t}-v \triangle u+u \cdot \nabla u+\alpha \theta g+\nabla p=0 \\
\operatorname{div}(u)=0 \\
\theta_{t}-\kappa \Delta \theta+u \cdot \nabla \theta=0
\end{array}\right.
$$

The global well-posedness of (2) with $v>0, \kappa>0$ is well-known (see [3]). However, for the case of $v=0$ and $\kappa=0$ in (2), the global existence of solution is still an outstanding open problem in the mathematical fluid mechanics (see [4-6]). Recently, some authors obtain the global well-posedness of (2) with partial viscosity cases (i.e., either the zero diffusivity case: $\kappa=0$ and $v>0$, or the zero viscosity case: $\kappa>0$ and $v=0$ ) (see [6-12]).

Some attentions have been paid to the nonhomogeneous case (1). In [13], the authors investigated the initial-boundary problems of (1) and obtained the global well-posedness. 
In [14], they studied an optimum control problem of mathematical model describing steady non-isothermal creep of incompressible fluid through local Lipschitz bounded region. In [15], they studied an optimal control problem for the mathematical model that describes steady non-isothermal creeping flows of an incompressible fluid through a locally Lipschitz bounded domain. In [16], the initial-boundary value problem of completely incompressible Navier-Stokes equations with viscosity coefficient $v$ and heat conductivity $\mathcal{k}$ varying with temperature by the power law of Chapman-Enskog are studied. When $\kappa=0$, the method used in [16] is not applicable. We must seek new methods to overcome the difficulty.

The purpose of this paper is to study the well-posedness of the Boussinesq system (1). Equations (1) corresponds to the physical environment which we can't ignore the variation of fluid viscosity (and thermal conductivity) with temperature (for more details see [17] and the references therein). The existing literature has more discussion on the constant viscosity and less discussions on the viscosity of temperature. This paper will provide some methods for studying other problems when viscosity relates to temperature. In the present paper, we consider the system (1) without thermal conductivity, and with the viscosity $v$ dependent on $\theta$. The main difficulty is that we can not use the results obtained previously for the constant viscosity. We firstly use the method of iteration, then we transform the problem into a constant viscosity problem. This is the biggest innovation of this paper. Since the Besov space is more meticulous than the traditional Sobolev space, the results obtained in this paper are no longer correct in the Sobolev space. In the present paper, we study the following equations:

$$
\left\{\begin{array}{l}
u_{t}-\operatorname{div}(v(\theta) \nabla u)+u \cdot \nabla u+\alpha \theta g+\nabla p=0, \\
\operatorname{div}(u)=0, \\
\theta_{t}+u \cdot \nabla \theta=0, \\
t=0, u=u_{0}, \theta=\theta_{0} .
\end{array}\right.
$$

In order to have a clear idea of our purpose, we shall recall some research history for the following Navier-Stokes equations:

$$
\left\{\begin{array}{l}
\partial_{t} \rho+\nabla \cdot(\rho u)=0, \\
\partial_{t}(\rho u)+\nabla \cdot(\rho u \times u)-\mu \Delta u=\rho f, \\
\nabla \cdot u=0 .
\end{array}\right.
$$

In [18], Fujita \& Kato proved the global existence and uniqueness of problem (4) in the critical Sobolev space $\dot{H}^{\frac{N}{2}} \times\left(\dot{H}^{\frac{N}{2}-1}\right)^{N}$. Precisely, if $(\rho, u)$ is a solution of (4), with initial data $\left(\rho_{0}(x), u_{0}(x)\right)$, then:

$$
\rho_{\lambda}(t, x)=\rho\left(\lambda^{2} t, \lambda x\right), \quad u_{\lambda}(t, x)=\lambda u\left(\lambda^{2} t, \lambda x\right)
$$

is also the solution of (4) with initial data $\left(\rho_{0, \lambda}(x), u_{0, \lambda}(x)\right)=\left(\rho_{0}(\lambda x), \lambda u_{0}(\lambda x)\right)$.

Subsequently, in [19], Danchin generalized the results by Fujita \& Kato [18] in Besov space $\left(\dot{B}_{2,1}^{\frac{N}{2}-1}\right)^{N}$; see also [20-24]. Some ideas of this paper came from $[19,20]$. Some new results about the equations may be found in [25-30].

We suppose that the initial data $\theta_{0}>0$. In the present paper, we shall establish the well-posedness of the non-homogeneous Bounssinesq Equation (3) in $\dot{B}_{p, 1}^{N / p}$ (see the definition in Section 2). Since the Besov spaces are symmetry, the results obtained in this paper have the property of symmetry. We shall restrict our work to solutions such that the temperature $\theta$ is a small perturbation of a constant temperature $\bar{\theta}$. As we know, there are inevitable errors in the process of modeling or measurement. We are looking forward to understanding the impact of these errors on the behavior of the solutions. This paper solves this problem well. There are few relevant studies at present. Without loss of generality, in 
the following, we take $\bar{\theta}=1, v \in C^{\infty}$ and $v(\bar{\theta})=v(1)=\underline{v}$. Therefore, Equation (3) can be rewritten as:

$$
\left\{\begin{array}{l}
u_{t}-\underline{v} \Delta u+u \cdot \nabla u+\nabla p=G, \\
\operatorname{div}(u)=0 \\
\theta_{t}+u \cdot \nabla \theta=0 \\
t=0, u=u_{0}, \theta=\theta_{0}
\end{array}\right.
$$

where,

$$
G=\nabla \cdot[(v(\theta)-\underline{v}) \nabla u]-\alpha \theta g .
$$

We write:

$$
\widetilde{v}(\theta+1)=v(\theta) \text { and } \widetilde{v}(\underline{\theta})=1 .
$$

Let us now state our main results.

Theorem 1. Let $1<p<2 N$, then for $\left(\theta_{0}, u_{0}\right) \in\left(\dot{B}_{p, 1}^{N / p}\right) \times\left(\dot{B}_{p, 1}^{N / p-1}\right)^{N}, g \in L_{T}^{1}\left(\dot{B}_{p, 1}^{N / p-1}\right)$ $\cap L_{T}^{2}\left(\dot{B}_{p, 1}^{N / p}\right)$ there exists $T\left(\theta_{0}, u_{0}\right)>0$, such that the problem (5) admits a solution $(\theta, u)$ with:

$$
\begin{gathered}
\theta \in C\left([0, T), \dot{B}_{p, 1}^{N / p}\right) \bigcap L_{T}^{\infty}\left(\dot{B}_{p, 1}^{N / p}\right), \\
u \in C\left([0, T), \dot{B}_{p, 1}^{N / p-1}\right) \bigcap L_{T}^{\infty}\left(\dot{B}_{p, 1}^{N / p-1}\right) \bigcap L_{T}^{1}\left(\dot{B}_{p, 1}^{N / p+1}\right) .
\end{gathered}
$$

Moreover, if there exist a small constant $\varepsilon$, such that:

$$
\left\|u_{0}\right\|_{L_{T}^{\infty}\left(\dot{B}_{p, 1}^{N / p-1}\right)}+\|g\|_{L_{T}^{1}\left(\dot{B}_{p, 1}^{N / p-1}\right)} \leq \varepsilon \underline{\mu},
$$

then $T=+\infty$. If $1<p \leq N$, the solution is unique.

The present paper is structured as follows: in the next section, we show some preliminaries. In Section 3, we show the existence of the solution. The uniqueness is presented in Section 4. Some conclusions are included in Section 5.

Remark 1. Throughout this paper, $C$ stands for a 'harmless' uniform constant, and we sometimes use the notation $A \lesssim B$ as an equivalent of $A \leq C B$. The notation $A \approx B$ means that $A \lesssim B$ and $B \lesssim A$.

\section{Some Results on Besov Spaces}

\subsection{Littlewood-Paley Theory}

At the beginning, we shall recall the Littlewood-Paley decomposition.

Take $\chi, \phi \in C^{\infty}\left(R^{N}\right)$ supported on $B=\left\{\xi \in R^{N},|\xi| \leq 4 / 3\right\}$ and $\Gamma=\left\{\xi \in R^{N}, 3 / 4 \leq\right.$ $|\xi| \leq 8 / 3\}$ respectively, such that:

$$
\sum_{j \in \mathbb{Z}} \phi\left(2^{-j} \xi\right)=1, \quad \chi=1-\sum_{j \geq 0} \phi\left(2^{-j} \xi\right), \quad \forall \xi \neq 0 .
$$

Denoting:

$$
\Delta_{j} u=F^{-1}\left(\phi\left(2^{-j} \cdot\right) \hat{u}(\cdot)\right)=2^{N j} \int_{R^{N}} \psi\left(2^{j} y\right) u(x-y) d y, \quad \text { for } j \in \mathbb{Z},
$$

and:

$$
S_{j} u=\sum_{k \leq j-1} \Delta_{k} u=2^{N j} \int_{R^{N}} \psi_{1}\left(2^{j} y\right) u(x-y) d y,
$$


where $\hat{u}=\mathcal{F}(u)$ denote the Fourier transformation of $\mathbf{u}, \psi=\mathcal{F}^{-1}(\phi(\cdot))$, and $\psi_{1}=$ $\mathcal{F}^{-1}(\chi(\cdot))$. The formal decomposition:

$$
u=\sum_{j=-\infty}^{\infty} \Delta_{j} u,
$$

is called homogenous Littlewood-Paley decomposition. This dyadic decomposition has a nice quasi-orthogonality, and we have:

$$
\begin{gathered}
\Delta_{i} \Delta_{j} u \equiv 0, \quad \text { if, } \quad|i-j| \geq 2, \quad \text { and } \\
\Delta_{i}\left(S_{j-1} u \Delta_{j} u\right) \equiv 0, \quad \text { if, } \quad|i-j| \geq 5 .
\end{gathered}
$$

The details of Littlewood-Paley decomposition can be found in [31,32].

\subsection{The Homogeneous Besov Spaces}

In the following, we shall define the functional spaces in which we shall work in.

Definition 1. For $s \in R,(p, r) \in[1,+\infty] \times[1,+\infty]$, and $u \in \mathcal{S}^{\prime}\left(R^{N}\right)$. Define:

$$
\dot{B}_{p, r}^{s}=\left\{u \in \mathcal{S}^{\prime}\left(R^{N}\right),\|u\|_{\dot{B}_{p, r}^{s}}<+\infty\right\},
$$

where:

$$
\|u\|_{\dot{B}_{p, r}^{s}}=\left\{\begin{array}{l}
\left(\sum_{j \in \mathbb{Z}} 2^{r j s}\left\|\Delta_{j} u\right\|_{L^{p}}^{r}\right)^{\frac{1}{r}}, \quad r<+\infty, \\
\sup _{j} 2^{j s}\left\|\Delta_{j} u\right\|_{L^{p}}, \quad r=+\infty .
\end{array}\right.
$$

Let us now recall some classical properties for these Besov spaces (see [23,24])

Proposition 1. The following properties hold:

(i) There exists a uniform constant $C$, such that,

$$
C^{-1}\|u\|_{\dot{B}_{p, r}^{s}} \leq\|\nabla u\|_{\dot{B}_{p, r}^{s-1}} \leq C\|u\|_{\dot{B}_{p, r}^{s}},
$$

(ii) Sobolev embedding: for $p_{1} \leq p_{2}$ and $r_{1} \leq r_{2}$, then,

$$
\dot{B}_{p_{1}, r_{1}}^{s} \hookrightarrow \dot{B}_{p_{2}, r_{2}}^{s-N\left(\frac{1}{p_{1}}-\frac{1}{p_{2}}\right)},
$$

(iii) For $s>0, \dot{B}_{p, r}^{s} \cap L^{\infty}$ is an algebra. Moreover, for any $p \in[1,+\infty]$, then,

$$
\dot{B}_{p, 1}^{N / p} \hookrightarrow \dot{B}_{p, \infty}^{N / p} \bigcap L^{\infty} .
$$

(iv) Interpolation: $\left[\dot{B}_{p, r}^{s_{1}}, \dot{B}_{p, r}^{s_{2}}\right]_{\theta, r^{\prime}}=\dot{B}_{p, r^{\prime}}^{\theta s_{1}+(1-\theta) s_{2}}$.

Through this paper, we shall use the product law in Besov spaces. These product laws are proved in [20,33].

Proposition 2. Let $\left(p, p_{1}, p_{2}\right) \in[1,+\infty]^{3}$ such that:

$$
\frac{1}{p} \leq \frac{1}{p_{1}}+\frac{1}{p_{2}}
$$

We get: 
(i) If:

$$
s_{1}+s_{2}+\operatorname{Ninf}\left(0,1-\frac{1}{p_{1}}-\frac{1}{p_{2}}\right)>0, \quad s_{1}<\frac{N}{p_{1}} \quad \text { and } \quad s_{2}<\frac{N}{p_{2}},
$$

there holds,

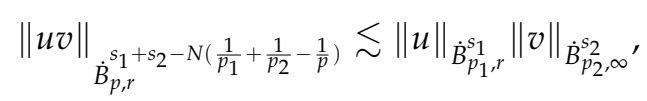

furthermore, if $s_{1}=\frac{N}{p_{1}}$ and $s_{2}=\frac{N}{p_{2}}$, we take $r=1$.

(ii) If $|s|<\left|\frac{N}{p}\right|$ and $p \geq 2$, then we get:

$$
\|u v\|_{\dot{B}_{p, r}^{s}} \lesssim\|u\|_{\dot{B}_{p, r}^{s}}\|v\|_{\dot{B}_{p, \infty}^{N / p} \cap L^{\infty}} .
$$

(iii) If $s_{1}+s_{2}=0, s_{1} \in\left(-\frac{N}{p_{1}}, \frac{N}{p_{1}}\right]$ and $\frac{1}{p_{1}}+\frac{1}{p_{2}} \leq 1$, then:

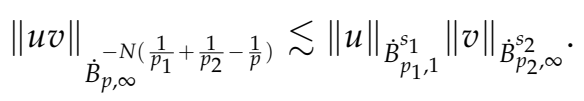

Additionally, we need the definition of $\tilde{L}_{T}^{\alpha}\left(\dot{B}_{p, r}^{s}\right)$ introduced in $[19,20,31]$.

Definition 2. Let $(r, \alpha, p) \in[1,+\infty]^{3}, T \in[0,+\infty]$ and $s \in R$. We set:

$$
\|u\|_{\tilde{L}_{T}^{\alpha}\left(\dot{B}_{p, r}^{s}\right)} \triangleq\left(\sum_{j \in \mathbb{Z}} 2^{j r s}\left(\int_{0}^{T}\left\|\Delta_{j} u(t)\right\|_{L^{p}}^{\alpha} d t\right)^{r / \alpha}\right)^{1 / r} .
$$

By virtue of the Minkowski inequality, we get:

$$
\|u\|_{\tilde{L}_{T}^{\alpha}\left(\dot{B}_{p, r}^{s}\right)} \leq\|u\|_{L_{T}^{\alpha}\left(\dot{B}_{p, r}^{\alpha}\right)}, \quad \text { if } \alpha \leq r .
$$

Thus,

$$
\|u\|_{L_{T}^{\alpha}\left(\dot{B}_{p, r}^{s}\right)} \leq\|u\|_{\tilde{L}_{T}^{\alpha}\left(\dot{B}_{p, r}^{s}\right)}, \quad \text { if } r \leq \alpha .
$$

Moreover, for $\theta \in(0,1]$, we have:

$$
\|u\|_{\tilde{L}_{T}^{\alpha}\left(\dot{B}_{p, r}^{s}\right)} \leq\|u\|_{\tilde{L}_{T}^{\alpha_{1}}\left(\dot{B}_{p, r}^{s_{1}}\right)}^{\dot{s}_{1}}\|u\|_{\tilde{L}_{T}^{\alpha_{2}}\left(\dot{B}_{p, r}^{s_{2}}\right)^{\prime}}^{1-\theta}
$$

with,

$$
\frac{1}{\alpha}=\frac{\theta}{\alpha_{1}}+\frac{1-\theta}{\alpha_{2}}, \text { and } \quad s=\theta s_{1}+(1-\theta) s_{2} .
$$

\subsection{Estimates for Linear Transport Equation}

In the following, we recall some estimates for the following linear transport equation:

$$
\left\{\begin{array}{l}
\partial_{t} g+\nabla \cdot(v g)=F \\
g(0, x)=g_{0}
\end{array}\right.
$$

The following results will hold, (see proof in $[19,24,33]$ ).

Proposition 3. Let $(p, r) \in[1,+\infty]^{2}$ and s be such that $-1-\operatorname{Ninf}\left(\frac{1}{p^{\prime}}, \frac{1}{p}\right)<s<1+\frac{N}{p}$ where $p^{\prime}$ is the conjugate of $p$. Let $v$ be a free divergence vector such that $\nabla v \in L^{1}\left(0, T ; \dot{B}_{p, r}^{N / p} \cap L^{\infty}\right)$. Suppose that $g_{0} \in \dot{B}_{p, r}^{s}$ and $F \in L^{1}\left(0, T, \dot{B}_{p, r}^{s}\right)$, and $g$ be a solution of (21) then holds:

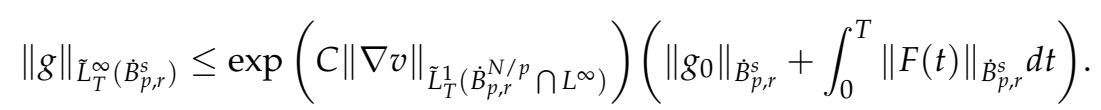


Proposition 4. Let $p \in(1,+\infty)$ and $-1-\operatorname{Ninf}\left(\frac{1}{p}, \frac{1}{p^{\prime}}\right)<s<\frac{N}{p}$, where $p^{\prime}$ is the conjugate exponent of $p$. Let $u_{0} \in \dot{B}_{p, r}^{s}, F \in \tilde{L}_{T}^{1}\left(\dot{B}_{p, r}^{s}\right)$ and $v$ be a free divergence vector such that $\nabla v \in$ $L^{1}\left(0, T ; \dot{B}_{p, r}^{N / p} \cap L^{\infty}\right)$, then Let $u$ be a solution of the following system:

$$
\left\{\begin{array}{l}
\partial_{t} u+v \cdot \nabla u-v \Delta u+\nabla P=F \\
\nabla \cdot u=0 \\
u(0, x)=u_{0}
\end{array}\right.
$$

where $v$ is a positive constant. Then there exists a constant $C$ such that the following estimates hold:

$$
\begin{aligned}
&\|u\|_{\tilde{L}_{T}^{\infty}\left(\dot{B}_{p, r}^{s}\right)}+v\|u\|_{\tilde{L}_{T}^{1}\left(\dot{B}_{p, r}^{s+2}\right)}+\|\nabla P\|_{\tilde{L}_{T}^{1}\left(\dot{B}_{p, r}^{s}\right)} \\
& \leq \exp \left(C\|\nabla v\|_{\tilde{L}_{T}^{1}\left(\dot{B}_{p, r}^{N / p} \cap L^{\infty}\right)}\right)\left(\left\|u_{0}\right\|_{\dot{B}_{p, r}^{s}}+C\|F\|_{\tilde{L}_{T}^{1}\left(\dot{B}_{p, r}^{s}\right)}\right) .
\end{aligned}
$$

\section{The Existence of the Solution}

In this section we shall prove the existence of the solution for (5). We state the results as following.

Theorem 2. Let $1<p<2 N$, then for $\left(\theta_{0}, u_{0}\right) \in\left(\dot{B}_{p, 1}^{N / p}\right) \times\left(\dot{B}_{p, 1}^{N / p-1}\right)^{N}, g \in L_{T}^{1}\left(\dot{B}_{p, 1}^{N / p-1}\right)$ $\cap L_{T}^{2}\left(\dot{B}_{p, 1}^{N / p}\right)$ there exists $T\left(\theta_{0}, u_{0}\right)>0$, such that the problem (5) admits a solution $(\theta, u)$ with:

$$
\begin{gathered}
\theta \in C\left([0, T), \dot{B}_{p, 1}^{N / p}\right) \bigcap L_{T}^{\infty}\left(\dot{B}_{p, 1}^{N / p}\right), \\
u \in C\left([0, T), \dot{B}_{p, 1}^{N / p-1}\right) \bigcap L_{T}^{\infty}\left(\dot{B}_{p, 1}^{N / p-1}\right) \bigcap L_{T}^{1}\left(\dot{B}_{p, 1}^{N / p+1}\right) .
\end{gathered}
$$

Moreover, if there exist a small constant $\varepsilon$, such that:

$$
\left\|u_{0}\right\|_{L_{T}^{\infty}\left(\dot{B}_{p, 1}^{N / p-1}\right)}+\|g\|_{L_{T}^{1} \dot{B}_{p, 1}^{N / p-1}} \leq \varepsilon \underline{\mu},
$$

then $T=+\infty$.

Proof. We shall prove this results by iteration. Denoting,

$$
\sum_{j \leq n} \Delta_{j} \theta=\theta^{n}, \quad \sum_{j \leq n} \Delta_{j} u=u^{n}, \quad \sum_{j \leq n} \Delta_{j} p=p^{n} .
$$

We shall build an approximate smooth solution $\left(\theta^{n}, u^{n}, p^{n}\right)$ of (5) satisfying,

$$
\left\{\begin{array}{l}
\partial_{t} \theta^{n+1}+u^{n} \cdot \nabla \theta^{n+1}=0, \\
\partial_{t} u^{n+1}+u^{n} \cdot \nabla u^{n+1}-\underline{v} \Delta u^{n+1}+\nabla p^{n+1}=G^{n}, \\
\nabla \cdot u^{n+1}=0 \\
\left(\theta^{1}, u^{1}\right)=S_{2}\left(\theta_{0}, u_{0}\right) \\
\left.\left(\tau^{n+1}, u^{n+1}\right)\right|_{t=0}=S_{n+2}\left(\theta_{0}, u_{0}\right),
\end{array}\right.
$$

where,

$$
G^{n}=\nabla \cdot\left[\left(\widetilde{v}\left(\theta^{n}+1\right)-\underline{v}\right) \nabla u^{n}\right]-\alpha \theta^{n} g .
$$

Obviously, from Propositions 3 and 4, we know that there exist a $T$ such that (25) admits a unique smooth solution in $t \in[0, T]$. Then, the proof of Theorem 2 is divided into two steps:

(1) The uniform a priori estimates for $\left(\theta^{n}, u^{n}\right)$. 
(2) The proof of the convergence of the sequences.

We begin to obtain the uniform estimates for $\left(\theta^{n}, u^{n}\right)$

Denoting:

$$
I_{0} \triangleq\left\|\theta_{0}\right\|_{\dot{B}_{p, 1}^{N / p}}+\left\|u_{0}\right\|_{\dot{B}_{p, 1}^{N / p-1}}
$$

and,

$$
E_{T}^{n}=\left\|\theta^{n}\right\|_{\tilde{L}_{T}^{\infty}\left(\dot{B}_{p, 1}^{N / p}\right)}+\left\|u^{n}\right\|_{\tilde{L}_{T}^{\infty}\left(\dot{B}_{p, 1}^{N / p-1}\right)}+\underline{\mu}\left\|u^{n}\right\|_{\tilde{L}_{T}^{1}\left(\dot{B}_{p, 1}^{N / p+1}\right)} .
$$

Let:

$$
\chi_{T}=\tilde{L}_{T}^{\infty}\left(\dot{B}_{p, 1}^{N / p}\right) \times\left(\tilde{L}_{T}^{\infty}\left(\dot{B}_{p, 1}^{N / p-1}\right) \bigcap \tilde{L}_{T}^{1}\left(\dot{B}_{p, 1}^{N / p+1}\right)\right)^{N} .
$$

Now, we shall prove that $\left\{\left(\theta^{n}, u^{n}\right)_{n \in \mathbb{Z}}\right\}$ is uniformly bounded in $\chi_{T}$. Moreover, $\forall n \in \mathbb{Z}$, we have the following conclusion:

$$
\text { claim: } \quad E_{T}^{n} \leq 4 I_{0} .
$$

We shall prove these by induction. For $n=0$, they are valid obviously. We assume that for a fixed $n,\left(\theta^{n}, u^{n}\right) \in \chi_{T}$ is valid and the claim holds, we shall show that for $n+1$, $\left(\theta^{n+1}, u^{n+1}\right) \in \chi_{T}$ and the claim are also valid.

From (25), by Propositions 3 and 4, we have:

$$
\left\|\theta^{n+1}\right\|_{\tilde{L}_{T}^{\infty}\left(\dot{B}_{p, 1}^{N / p}\right)} \leq e^{\int_{0}^{T} c\left\|\nabla u^{n}\right\|_{\dot{B}_{p, 1}^{N / 1}} d t}\left\|\theta_{0}^{n+1}\right\|_{\dot{B}_{p, 1}^{N / p}}
$$

and:

$$
\begin{aligned}
\left\|u^{n+1}\right\|_{\tilde{L}_{T}^{\infty}\left(\dot{B}_{p, 1}^{N / p-1}\right)}+\underline{v}\left\|u^{n+1}\right\|_{\tilde{L}_{T}^{1}\left(\dot{B}_{p, 1}^{N / p+1}\right)}+\left\|\nabla p^{n}\right\|_{\tilde{L}_{T}^{1}\left(\dot{B}_{p, 1}^{N / p-1}\right)} & \\
& \leq e^{\int_{0}^{T} c\left\|\nabla u^{n}\right\|_{\dot{B}_{p, 1}^{N / p}} d t}\left(\int_{0}^{T}\left\|G^{n}\right\|_{\dot{B}_{p, 1}^{N / p-1}} d t+\left\|u_{0}\right\|_{\dot{B}_{p, 1}^{N / p-1}}\right) .
\end{aligned}
$$

By the induction hypothesis, taking $T_{1}<T$ small enough, such that:

$$
e^{c\left\|u^{n}\right\|_{\tilde{L}_{T_{1}}^{1}\left(B_{p, 1}^{N / p+1}\right)}} \leq 2
$$

then, we obtain:

$$
\left\|\theta^{n+1}\right\|_{\tilde{L}_{T_{1}}^{\infty}\left(\dot{B}_{p, 1}^{N / p}\right)} \leq 2\left\|\theta_{0}\right\|_{\dot{B}_{p, 1}^{N / p}}
$$

From (30) and (31), we have:

$$
\begin{aligned}
\left\|u^{n+1}\right\|_{\tilde{L}_{T_{1}}^{\infty}\left(\dot{B}_{p, 1}^{N / p-1}\right)}+\underline{v}\left\|u^{n+1}\right\|_{\tilde{L}_{T_{1}}^{1}\left(\dot{B}_{p, 1}^{N / p+1}\right)}+ & \left\|\nabla p^{n}\right\|_{\tilde{L}_{T_{1}}^{1}\left(\dot{B}_{p, 1}^{N / p-1}\right)} \\
& \leq 2\left(\int_{0}^{T_{1}}\left\|G^{n}\right\|_{\dot{B}_{p, 1}^{N / p-1}} d t+\left\|u_{0}\right\|_{\dot{B}_{p, 1}^{N / p-1}}\right) .
\end{aligned}
$$

We now want to deal with $\int_{0}^{T_{1}}\left\|G^{n}\right\|_{\dot{B}_{p, 1}^{N / p-1}} d t$. Owing to Taylor's formula and Proposition 2, for $1<p<2 N$, we obtain:

$$
\begin{array}{ll}
\left\|\nabla \cdot\left[\left(\widetilde{v}\left(\theta^{n}+1\right)-\underline{v}\right) \nabla u^{n}\right]\right\|_{\tilde{L}_{T_{1}}^{1}\left(\dot{B}_{p, 1}^{N / p-1}\right)} & \\
\left\|\left[\left(\widetilde{v}\left(\theta^{n}+1\right)-\underline{v}\right) \nabla u^{n}\right]\right\|_{\tilde{L}_{T_{1}}^{1}\left(\dot{B}_{p, 1}^{N / p}\right)} & \\
& \lesssim\left(\left\|\theta^{n}\right\|_{\tilde{L}_{T_{1}}^{\infty}\left(\dot{B}_{p, 1}^{N / p}\right)}\left\|u^{n}\right\|_{\tilde{L}_{T_{1}}^{1}\left(\dot{B}_{p, 1}^{N / p+1}\right)} .\right.
\end{array}
$$


Combining (26) and (34), and using (15), we can get:

$$
\begin{aligned}
\left\|G^{n}\right\|_{\tilde{L}_{T_{1}}^{1}\left(\dot{B}_{p, 1}^{N / p-1}\right)} \lesssim\left\|\theta^{n}\right\|_{\tilde{L}_{T_{1}}^{\infty}\left(\dot{B}_{p, 1}^{N / p}\right)}\left\|u^{n}\right\|_{\tilde{L}_{T_{1}}^{1}\left(\dot{B}_{p, 1}^{N / p+1}\right)}+\alpha\left\|\theta^{n}\right\|_{\tilde{L}_{T_{1}}^{\infty}\left(\dot{B}_{p, 1}^{N / p}\right)}\|g\|_{\tilde{L}_{T_{1}}^{1}\left(\dot{B}_{p, 1}^{N / p-1}\right)} \\
\lesssim 4 I_{0}\left\|u^{n}\right\|_{\tilde{L}_{T_{1}}^{1}\left(\dot{B}_{p, 1}^{N / p+1}\right)}+\alpha I_{0}\|g\|_{\tilde{L}_{T_{1}}^{1}\left(\dot{B}_{p, 1}^{N / p-1}\right)} . \\
.
\end{aligned}
$$

Therefore, by the induction hypothesis, taking $T_{1}$ small enough, such that:

$$
4 I_{0}\left\|u^{n}\right\|_{\tilde{L}_{T_{1}}^{1}\left(\dot{B}_{p, 1}^{N / p+1}\right)}+\alpha I_{0}\|g\|_{\tilde{L}_{T_{1}}^{1}\left(\dot{B}_{p, 1}^{N / p-1}\right)} \leq I_{0}
$$

Then, from (32), (33), (35), and (36), we proved the claim that:

$$
\left(\tau^{\mathrm{n}+1}, \mathbf{u}^{\mathrm{n}+1}\right) \in \mathrm{X}_{\mathrm{T}_{1}} .
$$

Repeating the progress above, we see that if there exists a constant $\varepsilon$ small enough such that:

$$
\left\|u_{0}\right\|_{L_{T}^{\infty}\left(\dot{B}_{p, 1}^{N / p-1}\right)}+\|g\|_{\tilde{L}_{T}^{1}\left(\dot{B}_{p, 1}^{N / p-1}\right)} \leq \varepsilon \underline{v},
$$

then, the results presented above will be valid globally. (37) will be valid for all $T$. Thus, we have proved the claim (28).

We begin to get the convergence of the sequences.

To verify the convergence of the sequences of $\left(\theta^{n}, u^{n}\right)$, we shall consider the time derivative of the solution. We first show the following Lemma.

Lemma 1. Let $0<\eta<\inf \left(1, \frac{2 N}{p}\right), 1<p<2 N$, be such that $1+\eta<\frac{2 N}{p}$. Then $\nabla p^{n}$ is uniformly bounded in $L_{T}^{\frac{2}{2-\eta}}\left(\dot{B}_{p, 1}^{\frac{N}{p}-1-\eta}\right)$.

Proof. Since $g \in L_{T}^{1}\left(\dot{B}_{p, 1}^{N / p-1}\right) \cap L_{T}^{2}\left(\dot{B}_{p, 1}^{N / p}\right)$,we can easily get $g \in L_{T}^{\frac{2}{2-\eta}}\left(\dot{B}_{p, 1}^{N / p-1-\eta}\right)$ by interpolation.

Applying $\nabla \cdot$ on the second equation of (25), noting $\nabla \cdot u^{n}=0$, then we get:

$\left.\nabla \cdot\left(\nabla p^{n+1}\right)=\nabla \cdot\left[\nabla \cdot\left[\widetilde{v}\left(\theta^{n}+1\right)-\underline{v}\right) \nabla u^{n}\right]\right]+\nabla \cdot\left(\underline{v} \Delta u^{n+1}\right)-\nabla \cdot\left[u^{n} \cdot \nabla u^{n+1}\right]+\alpha \nabla \cdot\left[\theta^{n} g\right]$.

By the first step we have proved that:

$$
u^{n} \in\left(\tilde{L}_{T}^{\infty}\left(\dot{B}_{p, 1}^{N / p-1}\right) \bigcap \tilde{L}_{T}^{1}\left(\dot{B}_{p, 1}^{N / p+1}\right)\right)^{N},
$$

From (19) and (20), we get $u^{n} \in L_{T}^{2}\left(\dot{B}_{p, 1}^{N / p}\right)$ by interpolation. Similarly, we also have: $u^{n} \in L_{T}^{\frac{2}{2-\eta}}\left(\dot{B}_{p, 1}^{\frac{N}{p}+1-\eta}\right) ; u^{n} \in L_{T}^{\frac{2}{1-\eta}}\left(\dot{B}_{p, 1}^{\frac{N}{p}-\eta}\right)$ with $0<\eta<\inf \left(1, \frac{2 N}{p}\right)$.

By Taylor's formula, we obtain that:

$$
\begin{aligned}
& \left.\left\|\nabla \cdot \nabla \cdot\left[\left(\widetilde{v}\left(\theta^{n}+1\right)-\underline{v}\right) \nabla u^{n}\right]\right\|_{L_{T}^{2-\eta}\left(\dot{B}_{p, 1}^{p}-2-\eta\right.}\right) \\
& \lesssim \| \nabla \cdot\left[\left(\widetilde{v}\left(\theta^{n}+1\right)-\underline{v}\right) \nabla u^{n} \|_{L_{T}^{2-\eta}\left(\dot{B}_{p, 1}^{\frac{N}{p}-1-\eta}\right)}\right. \\
& \lesssim\left\|\left(\widetilde{v}\left(\theta^{n}+1\right)-\underline{v}\right) \nabla u^{n}\right\|_{L_{T}^{2-\eta}\left(\dot{B}_{p, 1}^{\frac{N}{p}-\eta}\right)}
\end{aligned}
$$

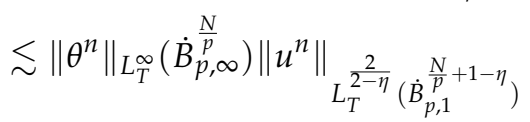

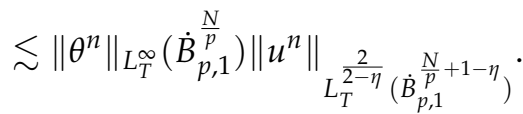


in deriving (39), we have used (14) and (15).

Since $u^{n+1} \in L_{T}^{\frac{2}{2-\eta}}\left(\dot{B}_{p, 1}^{\frac{N}{p}+1-\eta}\right)$, we can easily obtain:

$$
\Delta u^{n+1} \in L_{T}^{\frac{2}{2-\eta}}\left(\dot{B}_{p, 1}^{\frac{N}{p}-1-\eta}\right) .
$$

Using (14) and (15) we have:

$$
\begin{aligned}
& \left\|u^{n} \cdot \nabla u^{n+1}\right\|_{L_{T}^{\frac{2}{2-\eta}}\left(\dot{B}_{p, 1}^{\frac{N}{p}-1-\eta}\right)} \lesssim\left\|u^{n} \otimes u^{n+1}\right\|_{L_{T}^{2-\eta}\left(\dot{B}_{p, 1}^{\frac{N}{p}-\eta}\right)} \\
& \lesssim\left\|u^{n}\right\|_{L_{T}^{1-\eta}\left(\dot{B}_{p, 1}^{\frac{N}{p}-\eta}\right)}\left\|u^{n+1}\right\|_{L_{T}^{2}\left(\dot{B}_{p, 1}^{\frac{N}{p}}\right)} .
\end{aligned}
$$

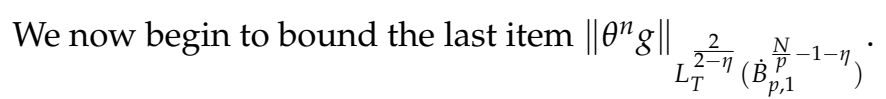

Using (15), we have:

$$
\left\|\theta^{n} g\right\|_{L_{T}^{2-\eta}\left(\dot{B}_{p, 1}^{\frac{N}{p}-1-\eta}\right)} \lesssim\left\|\theta^{n}\right\|_{L_{T}^{\infty}\left(\dot{B}_{p, 1}^{\frac{N}{p}}\right)}\|g\|_{L_{T}^{2-\eta}\left(\dot{B}_{p, 1}^{\frac{N}{p}-1-\eta}\right)} .
$$

Combining (38) and (42), we obtain the desired result.

In order to use the Ascoli Theorem, it suffices to estimate the derivatives of $\theta^{n}$ and $u^{n}$.

Proposition 5. For the sequence $\left(\theta^{n}, u^{n}\right)_{n \in N}$

(i) The sequence $\left(\partial_{t} \theta^{n}\right)_{n \in \mathbb{N}}$ is uniformly bounded in $L_{T}^{2}\left(\dot{B}_{p, 1}^{\frac{N}{p}-1}\right)$.

(ii) The sequence $\left(\partial_{t} u^{n}\right)_{n \in \mathbb{N}}$ is uniformly bounded in $L_{T}^{\frac{2}{2-\eta}}\left(\dot{B}_{p, 1}^{\frac{N}{p}-1-\eta}\right)$, for:

$$
0<\eta<\inf \left(1, \frac{2 N}{p}-1\right) \text { and } 1<p<2 N .
$$

Proof. From (25), we have:

$$
\partial_{t} \theta^{n+1}=-u^{n} \cdot \nabla \theta^{n+1} .
$$

Recall that $\left(\theta^{n+1}, u^{n}\right) \in L_{T}^{\infty}\left(\dot{B}_{p, 1}^{N / p}\right) \times\left(L_{T}^{2}\left(\dot{B}_{p, 1}^{N / p}\right)\right)^{N}$, from (43), we have:

$$
\partial_{t} \theta^{n+1} \in L_{T}^{2}\left(\dot{B}_{p, 1}^{N / p-1}\right) .
$$

Similarly, we get:

$$
\partial_{t} u^{n+1}=-u^{n} \cdot \nabla u^{n+1}+\underline{v} \Delta u^{n+1}-\nabla p^{n+1}+G^{n} .
$$

Then we get the desired result (ii) from Lemma 1.

Now we turn to the proof of the existence of the solution. According to Proposition 5, Cauchy-Schwarz inequality and Hölder's inequality, we deduce the following Corollary.

Corollary 1. For the sequence $\left(\theta^{n}, u^{n}\right)$ :

(i) The sequence $\left(\theta^{n}\right)_{n \in \mathbb{N}}$ is uniformly bounded in $C^{\frac{1}{2}}\left([0, T], \dot{B}_{p, 1}^{\frac{N}{p}-1}\right)$,

(ii) The sequence $\left(u^{n}\right)_{n \in \mathbb{N}}$ is uniformly bounded in $C^{\frac{\eta}{2}}\left(\dot{B}_{p, 1}^{\frac{N}{p}-1-\eta}\right)^{N}$, for,

$$
0<\eta<\inf \left(1, \frac{2 N}{p}-1\right) \text {. }
$$


According to Corollary 1 , the sequence $\left(\theta^{n}, u^{n}\right)_{n \in N}$ is uniformly bounded in $C^{\frac{1}{2}}([0, T]$, $\left.\dot{B}_{p, 1}^{\frac{N}{p}-1}\right) \times C^{\frac{\eta}{2}}\left(\dot{B}_{p, 1}^{\frac{N}{p}-1-\eta}\right)^{N}$, thus is uniformly bounded in $C\left([0, T], \dot{B}_{p, 1}^{\frac{N}{p}-1}\right) \times C\left([0, T], \dot{B}_{p, 1}^{\frac{N}{p}-1-\eta}\right)^{N}$. We recall that the injection of $\dot{B}_{p q, l o c}^{s+\varepsilon}$ in $\dot{B}_{p q, l o c}^{s}$ is compact for all $\varepsilon>0$. (See the proof in [34]). Using the uniform estimates and applying the Ascoli's Theorem, there exists a subsequence $\left(\theta^{n^{\prime}}, u^{n^{\prime}}\right)$, which converges to $(\theta, u)$. We gather that $(\theta, u)$ is a solution of $(25)$ belongs to:

$$
\left(C\left([0, T], \dot{B}_{p, 1}^{N / p}\right) \bigcap L_{T}^{\infty}\left(\dot{B}_{p, 1}^{N / p}\right)\right) \times\left(C\left([0, T], \dot{B}_{p, 1}^{N / p-1}\right) \bigcap L_{T}^{\infty}\left(\dot{B}_{p, 1}^{N / p-1}\right) \bigcap L_{T}^{1}\left(\dot{B}_{p, 1}^{N / p+1}\right)\right) .
$$

\section{The Uniqueness of the Solution}

In this section we shall prove the uniqueness of the solution for (5). We shall only establish the uniqueness when $p=N$, the case when $1<p<N$ follows by injection.

We state the results as following.

Theorem 3. Let $\left(\theta^{i}, u^{i}, p^{i}\right),(i=1,2)$ be two solutions solve (5) with the same initial data $\left(\theta_{0}, u_{0}\right)$. Assume that:

$$
\begin{gathered}
g \in L^{1}\left([0, T], \dot{B}_{N, 1}^{0}\right), \\
\theta^{i} \in C\left([0, T], \dot{B}_{N, 1}^{1}\right) \bigcap L^{\infty}\left([0, T], \dot{B}_{N, 1}^{1}\right), \\
u^{i} \in C\left([0, T], \dot{B}_{N, 1}^{0}\right) \bigcap L^{\infty}\left([0, T], \dot{B}_{N, 1}^{0}\right) \bigcap L^{1}\left([0, T], \dot{B}_{N, 1}^{2}\right), \\
\nabla p^{i} \in L^{1}\left([0, T], \dot{B}_{N, 1}^{0}\right),
\end{gathered}
$$

there exists a constant $\varepsilon$ small enough, if we have:

$$
\left\|\theta^{1}\right\|_{L_{T}^{\infty}\left(\dot{B}_{N, 1}^{1}\right)} \leq \varepsilon
$$

then $\left(\theta^{1}, u^{1}, \nabla p^{1}\right)=\left(\theta^{2}, u^{2}, \nabla p^{2}\right)$.

Proof. Let $\left(\theta^{i}, u^{i}, \nabla p^{i}\right)(i=1,2)$ be two solutions to the system (5), we denote:

$$
(\delta \theta, \delta u, \delta \nabla p)=\left(\theta^{1}-\theta^{2}, u^{1}-u^{2}, \nabla p^{1}-\nabla p^{2}\right),
$$

then we have:

$$
\left\{\begin{array}{l}
\partial_{t} \delta \theta+u^{2} \cdot \nabla \delta \theta+\delta u \nabla \theta^{1}=0, \\
\partial_{t} \delta u+u^{1} \cdot \nabla \delta u-\underline{v} \Delta \delta u+\nabla \delta p=K, \\
\nabla \cdot(\delta u)=0, \\
\left.(\delta \theta, \delta u)\right|_{t=0}=(0,0),
\end{array}\right.
$$

where,

$$
K=\nabla \cdot\left[\left(v\left(\theta^{1}\right)-\underline{v}\right) \nabla u^{1}\right]-\nabla \cdot\left[\left(v\left(\theta^{2}\right)-\underline{v}\right) \nabla u^{2}\right]+\delta u \cdot \nabla u^{2}+\delta \theta g .
$$

We shall prove the uniqueness in the space $\mathfrak{D}_{T}$ with:

$$
\begin{aligned}
\mathfrak{D}_{T}=\left(C\left([0, T], \dot{B}_{N, \infty}^{0}\right) \bigcap\right. & \left.L^{\infty}\left([0, T], \dot{B}_{N, \infty}^{0}\right)\right) \times\left(C\left([0, T], \dot{B}_{N, \infty}^{-1}\right)\right. \\
& \left.\bigcap L^{\infty}\left([0, T], \dot{B}_{N, \infty}^{-1}\right) \bigcap L^{1}\left([0, T], \dot{B}_{N, \infty}^{1}\right) \times L^{1}\left([0, T], \dot{B}_{N, \infty}^{-1}\right)\right) .
\end{aligned}
$$

Firstly,we have to state that $(\delta \theta, \delta u, \delta \nabla p) \in \mathfrak{D}_{T}$.

According to our assumption on $\left(\theta^{i}, u^{i}\right)$, the estimates of paraproduct yield $\partial_{t} \theta^{i} \in$ $L_{T}^{2}\left(\dot{B}_{N, 1}^{0}\right)$. Therefore $\bar{\theta}^{i}=\theta^{i}-\theta_{0}$ belongs to $C^{\frac{1}{2}}\left([0, T], \dot{B}_{N, 1}^{0}\right)$. Which clearly entails by embedding:

$$
\delta \theta \in C\left([0, T], \dot{B}_{N, 1}^{0}\right) .
$$


We now define:

$$
u^{i}=u_{L}+\bar{u}^{i}, \nabla p^{i}=\nabla p_{L}^{i}+\nabla \overline{p^{i}} .
$$

The quantities $u_{L}$ and $\nabla p_{L}$ are defined by the system below:

$$
\left\{\begin{array}{l}
\partial_{t} u_{L}-\underline{v} \Delta u+\nabla p_{L}=0, \\
\nabla \cdot u_{L}=0, \\
\left.u_{L}\right|_{t=0}=u_{0} .
\end{array}\right.
$$

Thanks to Proposition 2.6 above and Proposition 2.1 in [32], we have:

$$
u_{L} \in C\left([0, T], \dot{B}_{N, 1}^{0}\right) \bigcap L^{1}\left([0, T], \dot{B}_{N, 1}^{2}\right),
$$

and,

$$
\nabla p_{L} \in L^{1}\left([0, T], \dot{B}_{N, 1}^{0}\right)
$$

We obviously have $\left.\overline{u^{i}}\right|_{t=0}=0$ and $(\bar{u}, \nabla \bar{p})$ verify:

$$
\left\{\begin{array}{l}
\partial_{t} \bar{u}^{i}-\underline{v} \Delta \overline{u^{i}}+\nabla \overline{p^{i}}=K\left(\theta^{i}, u^{i}\right), \\
\nabla \cdot \overline{u^{i}}=0 \\
\left.\overline{u^{i}}\right|_{t=0}=u_{0},
\end{array}\right.
$$

where $K\left(\theta^{i}, u^{i}\right)=-u^{i} \cdot \nabla u^{i}+\nabla \cdot\left[\left(\widetilde{v}\left(\theta^{n}+1\right)-\underline{v}\right) \nabla u^{n}\right]-\alpha \theta^{n} g$.

The product and composition laws in Besov Spaces insure that $K\left(\theta^{i}, u^{i}\right)$ belongs to $L_{T}^{1}\left(\dot{B}_{N, 1}^{-1}\right)$, thus we can easily get $K\left(\theta^{i}, u^{i}\right)$ belongs to $L_{T}^{1}\left(\dot{B}_{N, \infty}^{-1}\right)$.

The Proposition 2.6 above and Proposition 2.1 in [32] yield:

$$
\bar{u}^{i} \in\left(C\left([0, T], \dot{B}_{N, \infty}^{-1}\right) \bigcap L^{\infty}\left([0, T], \dot{B}_{N, \infty}^{-1}\right) \bigcap L^{1}\left([0, T], \dot{B}_{N, \infty}^{1}\right),\right.
$$

and,

$$
\bar{\nabla} p^{i} \in L^{1}\left([0, T], \dot{B}_{N, \infty}^{-1}\right)
$$

Since

$$
\delta \theta=\left(\theta^{2}-\theta_{0}\right)-\left(\theta^{1}-\theta_{0}\right), \delta u=\bar{u}^{2}-\bar{u}^{1} \quad \text { and } \quad \nabla \delta p=\nabla \bar{p}^{2}-\nabla \overline{p^{1}},
$$

on combining the above discussions, we can conclude:

$$
(\delta \theta, \delta u, \delta \nabla p) \in \mathfrak{D}_{T} .
$$

To get the estimates of $\|(\delta \theta, \delta u, \delta \nabla p)\|_{\mathfrak{D}_{T}}$, by Proposition 4 , we have:

$$
\|\delta u\|_{\tilde{L}_{T}^{\infty}\left(\dot{B}_{N, \infty}^{-1}\right)}+\underline{v}\|\delta u\|_{\tilde{L}_{T}^{1}\left(\dot{B}_{N, \infty}^{1}\right)}+\|\delta \nabla p\|_{\tilde{L}_{T}^{1}\left(\dot{B}_{N, \infty}^{-1}\right)} \lesssim \exp \left(\int_{0}^{T}\left\|\nabla u^{1}\right\|_{\dot{B}_{N, \infty}^{1} \cap L^{\infty}} d t\right) \int_{0}^{T}\|K\|_{\dot{B}_{N, \infty}^{-1}} d t .
$$

Noting (53), and using Proposition 2, we have:

$$
\begin{aligned}
& \left\|\nabla \cdot\left[\left(\widetilde{v}\left(\theta^{1}\right)-\underline{v}\right) \nabla u^{1}\right]-\right\| \nabla \cdot\left[\left(\widetilde{v}\left(\theta^{2}\right)-\underline{v}\right) \nabla u^{2}\right] \|_{\tilde{L}_{T}^{1}\left(\dot{B}_{N, \infty}^{-1}\right)} \\
& \lesssim\left\|\nabla \cdot\left[\left(\widetilde{v}\left(\theta^{1}\right)-\underline{v}\right) \nabla u^{1}\right]-\nabla \cdot\left[\left(\widetilde{v}\left(\theta^{1}\right)-\underline{v}\right) \nabla u^{2}\right]\right\|_{\tilde{L}_{T}^{1}\left(\dot{B}_{N, \infty}^{-1}\right)} \\
& +\left\|\nabla \cdot\left[\left(\widetilde{v}\left(\theta^{1}\right)-\underline{v}\right) \nabla u^{2}\right]-\nabla \cdot\left[\left(\widetilde{v}\left(\theta^{2}\right)-\underline{v}\right) \nabla u^{2}\right]\right\|_{\tilde{L}_{T}^{1}\left(\dot{B}_{N, \infty}^{-1}\right)} \\
& \left.\quad \lesssim\left\|\theta^{1}\right\|_{\tilde{L}_{T}^{\infty}\left(\dot{B}_{N, 1}^{1}\right)}\|\delta u\|_{\tilde{L}_{T}^{1}\left(\dot{B}_{N, \infty}^{1}\right)}+\|\delta \theta\|_{\tilde{L}_{T}^{\infty}\left(\dot{B}_{N, \infty}^{0}\right)}\left\|u^{2}\right\|_{\tilde{L}_{T}^{1}\left(\dot{B}_{N, 1}^{2}\right)}\right)
\end{aligned}
$$

and,

$$
\left\|\delta u \cdot \nabla u^{2}\right\|_{\tilde{L}_{T}^{1}\left(\dot{B}_{N, \infty}^{-1}\right)} \lesssim\|\delta u\|_{\tilde{L}_{T}^{\infty}\left(\dot{B}_{N, \infty}^{-1}\right)}\left\|u^{2}\right\|_{\tilde{L}_{T}^{1}\left(\dot{B}_{N, 1}^{2}\right)} .
$$


Now, we shall estimate the term $\delta \theta$. By Proposition 3 ,

$$
\|\delta \theta\|_{\tilde{L}_{T}^{\infty}\left(\dot{B}_{N, \infty}^{0}\right)} \leq \exp \left(\int_{0}^{T}\left\|\nabla u^{2}\right\|_{\dot{B}_{N, \infty}^{1} \cap L^{\infty}} d t\right) \int_{0}^{T}\left\|\delta u \nabla \theta^{1}\right\|_{\dot{B}_{N, \infty}^{0}} d t,
$$

Using $(51)_{3}$ and (13), we get:

$$
\begin{aligned}
\int_{0}^{T}\left\|\delta u \nabla \theta^{1}\right\|_{\dot{B}_{N, \infty}^{0}} d t=\int_{0}^{T}\left\|\nabla \cdot\left(\delta u \theta^{1}\right)\right\|_{\dot{B}_{N, \infty}^{0}} d t & \\
& =\int_{0}^{T}\left\|\delta u \theta^{1}\right\|_{\dot{B}_{N, \infty}^{1}} d t \lesssim \int_{0}^{T}\left\|\delta u \theta^{1}\right\|_{\dot{B}_{N, 1}^{1}} d t
\end{aligned}
$$

If we choose $s_{1}=1, s_{2}=1, p_{1}=p_{2}=p=N$ in (15), we obtain:

$$
\int_{0}^{T}\left\|\delta u \theta^{1}\right\|_{\dot{B}_{N, 1}^{1}} d t \lesssim \int_{0}^{T}\|\delta u\|_{\dot{B}_{N, \infty}^{1}}\left\|\theta^{1}\right\|_{\dot{B}_{N, 1}^{1}} d t \lesssim\left\|\theta^{1}\right\|_{\tilde{L}_{T}^{\infty}\left(\dot{B}_{N, 1}^{1}\right)}\|\delta u\|_{\tilde{L}_{T}^{1}\left(\dot{B}_{N, \infty}^{1}\right)} .
$$

We now begin to bound $\int_{0}^{T} \alpha\|\delta \theta g\|_{\tilde{L}_{T}^{1}\left(\dot{B}_{N, \infty}^{-1}\right)}$,

$$
\int_{0}^{T} \alpha\|\delta \theta g\|_{\tilde{L}_{T}^{1}\left(\dot{B}_{N, \infty}^{-1}\right)} \lesssim \alpha\|\delta \theta\|_{\tilde{L}_{T}^{\infty}\left(\dot{B}_{N, \infty}^{0}\right)}\|g\|_{\tilde{L}_{T}^{1}\left(\dot{B}_{N, 1}^{0}\right)} .
$$

in deriving (60), we have used (17).

Write:

$$
\gamma(T) \triangleq\|\delta \theta\|_{\tilde{L}_{T}^{\infty}\left(\dot{B}_{N, \infty}^{0}\right)}+\|\delta u\|_{\tilde{L}_{T}^{\infty}\left(\dot{B}_{N, \infty}^{-1}\right)}+\underline{\mu}\|\delta u\|_{\tilde{L}_{T}^{1}\left(\dot{B}_{N, \infty}^{1}\right)}+\|\delta \nabla p\|_{\tilde{L}_{T}^{1}\left(\dot{B}_{N, \infty}^{-1}\right)} .
$$

Then from (54)-(60), we get:

$$
\begin{aligned}
\gamma(T) \lesssim\left\|\theta^{1}\right\|_{\tilde{L}_{T}^{\infty}\left(\dot{B}_{N, 1}^{1}\right)}\|\delta u\|_{\tilde{L}_{T}^{1}\left(\dot{B}_{N, \infty}^{1}\right)}+\|\delta \theta\|_{\tilde{L}_{T}^{\infty}\left(\dot{B}_{N, \infty}^{0}\right)}\left\|u^{2}\right\|_{\tilde{L}_{T}^{1}\left(\dot{B}_{N, 1}^{2}\right)} \\
\quad+\|\delta u\|_{\tilde{L}_{T}^{\infty}\left(\dot{B}_{N, \infty}^{-1}\right)}\left\|u^{2}\right\|_{\tilde{L}_{T}^{1}\left(\dot{B}_{N, 1}^{2}\right)}+\alpha\|\delta \theta\|_{\tilde{L}_{T}^{\infty}\left(\dot{B}_{N, \infty}^{0}\right)}\|g\|_{\tilde{L}_{T}^{1}\left(\dot{B}_{N, 1}^{0}\right)} .
\end{aligned}
$$

Taking $T$ small enough, such that for any small positive constant $\varepsilon_{0}$, we have:

$$
\left\|\nabla u^{2}\right\|_{\tilde{L}_{T}^{1}\left(\dot{B}_{N, 1}^{1}\right)}+\|g\|_{L_{T}^{1}\left(\dot{B}_{N, 1}^{0}\right)} \leq \frac{1}{2} \varepsilon_{0} .
$$

Thus, we have:

$$
\gamma(T) \leq \frac{1}{2} \gamma(T) .
$$

we get,

$$
\gamma(T) \equiv 0,
$$

which yields uniqueness of the solutions.

\section{Conclusions}

In this paper, we studied the Cauchy problem for non-homogeneous Boussinesq equations. We proved the global existence of the solution when the initial velocity are small with respect to the viscosity, as well as the initial temperature approaches a positive constant on the critical Besov spaces $(\theta, u) \in L_{T}^{\infty}\left(\dot{B}_{p, 1}^{N / p}\right) \times L_{T}^{\infty}\left(\dot{B}_{p, 1}^{N / p-1}\right) \cap L_{T}^{1}\left(\dot{B}_{p, 1}^{N / p+1}\right)$ with $1<p<2 N$. Furthermore, we proved the uniqueness for $1<p \leq N$. When $N \leq p \leq 2 N$, the uniqueness is difficult. We can't get any result following the method proposed in this paper. We will consider the uniqueness for $N \leq p \leq 2 N$ in the future. We can also obtain similar results for other fluid equations. 
Author Contributions: Writing-original draft, Y.L. and B.O. All authors have read and agreed to the published version of the manuscript.

Funding: This research was funded by Key projects of universities in Guangdong Province (NATURAL SCIENCE) (2019KZDXM042) and the Research Fundations of Guangzhou Huashang College (2021HSKT01,2020HSDS01).

Institutional Review Board Statement: Not applicable.

Informed Consent Statement: Not applicable.

Data Availability Statement: Not applicable.

Acknowledgments: The authors would like to deeply thank all the reviewers for their insightful and constructive comments.

Conflicts of Interest: The authors declare no conflict of interest.

\section{References}

1. Drazin, P.G.; Reid, W.H. Hydrodynamic Stability; Cambridge University Press: Cambridge, UK, 1981.

2. Majda, A. Introduction to PDEs and Waves for the Atmosphere and Ocean; Courant Lecture Notes in Mathematics; AMS/CIMS: Providence, RI, USA, 2003; Volume 9.

3. Cannon, J.R.; Benedetto, E.D. The Initial Problem for the Boussinesq Equations with Data in $L^{p}$; Lecture Note in Mathematics; Springer: Berlin, Germany, 1980; Volume 771, pp. 129-144.

4. Chae, D.; Nam, H.-S. Local existence and blow-up criterion for the Boussinesq equations. Proc. R. Soc. Edinb. 1997, 127A, 935-946. [CrossRef]

5. Chae, D.; Kim, S.-K.; Nam, H.-S. Local existence and blow-up criterion of Holder continuous solutions of the Boussinesq equations. Nagoya Math. J. 1999, 155, 58-80. [CrossRef]

6. Taniuchi, Y. A note on the blow-up criterion for the inviscid 2-D Boussinesq equations. Lect. Notes Pure Appl. Math. 2002, 223, 131-140.

7. Chae, D. Global regularity for the 2D Boussinesq equations with partial viscosity terms. Adv. Math. 2006, 203, 497-513. [CrossRef]

8. Danchin, R.; Paicu, M. Existence and uniqueness results for the Boussinesq system with data in Lorentz spaces. Physica D 2008, 237, 1444-1460. [CrossRef]

9. Danchin, R.; Paicu, M. Global well-posedness issues for the inviscid Boussinesq system with Yudovich's type data. Commun. Math. Phys. 2009, 290, 1-14. [CrossRef]

10. Hou, T.Y.; Li, C. Global well-posedness of the viscous Boussinesq equations. Discret. Contin. Dyn. Syst. 2005, 12, 1-12. [CrossRef]

11. Liu, X.; Wang, M.; Zhang, Z. Local Well-Posedness and Blowup Criterion of the Boussinesq Equations in Critical Besov Spaces. J. Math. Fluid Mech. 2010, 12, 280-292.

12. Abidi, H.; Hmidi, T. On the global well-posedness for Boussinesq system. J. Differ. Equ. 2007, 233, 199-220. [CrossRef]

13. Sebastian, A.L.; Jose, L.B. The initial value problem for a genneralized Boussinesq model. Nonlinear Anal. 1999, 36, 457-480.

14. Baranovskii, E.S.; Domnich, A.A. Model of a Nonuniformly Heated Viscous Flow through a Bounded Domain. Differ. Equ. 2020, 56, 304-314. [CrossRef]

15. Baranovskii, E.S.; Domnich, A.A.; Artemov, M.A. Optimal Boundary Control of Non-Isothermal Viscous Fluid Flow. Fluids 2019, 4, 133. [CrossRef]

16. Guo, Z.H.; Li, Q.Y. Global existence and large time behaviors of the solutions to the full incompressible Navier-Stokes equations with temperature-dependent coefficients. J. Differ. Equ. 2021, 274, 876-923. [CrossRef]

17. Lorca, S.A.; Boldrini, J.L. Stationary solutions for genneralized Boussinesq model. J. Differ. Equ. 1996, 124, 389-406. [CrossRef]

18. Fujita, H.; Kato, T. On the Navier-Stokes initial value problem I. Arch. Ration. Mech. Anal. 1964, 16, 269-315. [CrossRef]

19. Danchin, R. Global existence in critical spaces for compressible Navier-Stokes equations. Invent. Math. 2000, 141, 579-614. [CrossRef]

20. Abidi, H.; Paicu, M. Global Existence for the Magnetohydrodynamic system in critical spaces. Proc. R. Soc. Edinb. 2008, 138A, 447-476. [CrossRef]

21. Chen, Q.; Miao, C.; Zhang, Z. On the well-posedness for the viscous shallow water equations. SIAM J. Math. Anal. 2008, 40, 443-474. [CrossRef]

22. Danchin, R. Local theory in Critical spaces for compressible viscous and heat conductive gases. Commun. Partial Differ. Equ. 2001, 26, 1183-1233. [CrossRef]

23. Danchin, R. Global existence in critical spaces for flows of compressible viscous and heat-conductive gases. Arch. Ration. Mech. Anal. 2001, 160, 1-39. [CrossRef]

24. Danchin, R. Density-dependent incompressible viscous fluids in critical spaces. Proc. R. Soc. Edinb. 2003, 133A, 1311-1334. [CrossRef]

25. Deng, W.; Wu, J.; Zhang, P. Stability of Couette flow for 2D Boussinesq system with vertical dissipation. J. Funct. Anal. 2021, 281, 109255. [CrossRef] 
26. Qiu, H.; Du, Y.; Yao, Z. A note on the regularity criterion of the two-dimensional Newton CBoussinesq equations. Nonlinear Anal. Real World Appl. 2011, 12, 2012-2015. [CrossRef]

27. Qiu, H.; Du, Y.; Yao, Z. Blow-up criteria for 3D Boussinesq equations in the multiplier space. Commun. Nonlinear Sci. Numer. Simul. 2011, 16, 1820-1824. [CrossRef]

28. Qiu, H.; Du, Y.; Yao, Z. A blow-up criterion for 3D Boussinesq equations in Besov spaces. Nonlinear Anal. Theory Methods Appl. 2010, 73, 806-815. [CrossRef]

29. Chen, W.; Ikehata, R. The Cauchy problem for the Moore-Gibson-Thompson equation in the dissipative case. J. Differ. Equ. 2021, 292, 176-219. [CrossRef]

30. Chen, W.; Palmieri, A. Weakly coupled system of semilinear wave equations with distinct scale-invariant terms in the linear part. Z. Angew. Math. Phys. 2019, 70, 67. [CrossRef]

31. Chemin, J.-Y. Perfect Incompressible Fluids; Oxford University Press: New York, NY, USA, 1998.

32. Chemin, J.-Y. Théorèmes D'unicité Pour le Systèème de Navie-Stockes Tridimensionnel. J. Anal. 1999, 77, 27-50.

33. Abidi, H.; Paicu, M. Éxistence globale pour un fluide inhomogène. Ann. Inst. Fourier 2007, 57, 883-917. [CrossRef]

34. Runst, T.; Sickel, W. Sobolev spaces of fractional order, Nemytskii operators, and nonlinear partial differential equations. In Nonlinear Analysis and Applications; Walter de Wruyter: Berlin, Germany, 1996; Volume 3. 